\title{
Sample PAC-Learnability in Model Inference
}

\author{
S.H. Nienhuys-Cheng ${ }^{1}$ and M. Polman ${ }^{1,2}$ \\ 1 Dept. of Computer Science, Erasmus University of Rotterdam, \\ P.O.Box 1738, 3000 DR Rotterdam, The NetherLands. \\ 2 Tinbergen Institute.
}

\begin{abstract}
In this article, PAC-learning theory is applied to model inference, which concerns the problem of inferring theories from facts in first order logic. It is argued that uniform sample PAC-learnability cannot be expected with most of the 'interesting' model classes. Polynomial sample learnability can only be accomplished in classes of programs having a fixed maximum number of clauses. We have proved that the class of context free programs in a fixed maximum number of clauses with a fixed maximum number of literals is learnable from a polynomial number of examples. This is also proved for a more general class of programs.
\end{abstract}

\section{Introduction and Preliminaries of PAC-Learning}

In the field of machine learning, two important paradigms are PAC-learning [15] and Inductive Logic Programming (ILP) [9]. Both paradigms are concerned with learning from examples within some domain. In this paper we will try to apply some of the theory developed around the sample (PAC-)learnability of concept classes to classes of 'models' in first order logic, by considering least Herbrand models of logic programs as concepts in PAC-learning. Now, establishing results about the sample learnability of model classes (globally, the number of examples required to PAC-learn them) requires information about their so-called Vapnik-Chervonenkis dimension. Specifically, we want to find an upper bound on this dimension, following Lemma 1 (see below). This upper bound involves the number of equivalence classes of programs, i.e. groups of programs behaving identically, on the set of examples of some maximal length. As we will show, for certain classes this number can be estimated by counting programs of a specific form. We performed this method on two model classes, thus proving that they are PAC-learnable from a polynomial number of examples (polynomial sample learnable). An algorithm that actually learns these classes is given by a slight adaptation of an algorithm by Shapiro [14].

Besides polynomial sample learnability, there is another learnability criterion, called uniform sample learnability. This criterion is more stringent and we will show, by a property of prime numbers, that it is too strict for the really 'interesting' classes of logic programs.

In the rest of this section and Section 2 we will state the basic notions in both PAC-learning and model inference, necessary for the results presented later on. The definitions used in this section mostly come from [10].

Basic notions Let $\Sigma$ be some finite alphabet. Let $X$ be the domain of our interest, namely, a set of finite strings over $\Sigma$. A subset $f$ of $X$ is called a concept. A number of concepts with distinct features can be grouped in a class of concepts $\mathcal{F}$. 
Example. As an example, consider the class of the monotone monomials over the domain of the $(0,1)$-strings. These are concepts representable by boolean functions consisting of the conjunction of a number of boolean variables. A concept contains those and only those strings for which its corresponding monomial formula returns true (e.g. the concept of strings of length 4 corresponding to the formula $a_{1} \wedge a_{4}$ would be the set $\{1001,1011,1101,1111\})$.

An example for some concept $f$ is a pair $(\alpha, V)$, where $\alpha \in X$ and $V=1$ if $\alpha \in f$ and $V=0$ if $\alpha \notin f$. Finally, $X^{[n]}$ is the set of all domain elements of length at most $n$.

Probabilistic issues In the PAC-learning paradigm, we are concerned with finding, with tunable probability, a concept in a concept class that is tunably close to the 'intended' concept, using examples for the latter concept to guide our actions. More specifically, examples for some target concept $f$ in class $\mathcal{F}$ are generated according to some unknown probability distribution $P$ on $X^{[n]}$, for some given $n$, and fed to a learning algorithm. Eventually, the algorithm should output a concept $g \in F$, such that with probability at least $1-\delta, P(f \Delta g) \leq \varepsilon^{3}$, where $\delta$ and $\varepsilon$ (and $n$ ) are the input parameters of the algorithm. A formal definition of a PAC-algorithm is as follows:

Definition PAC-learning algorithm. A learning algorithm $A$ is a $P A C$-algorithm for a class of concepts $\mathcal{F}$ over $X$ if

1. A takes as input $\varepsilon, \delta>0$ and $n \in \mathbb{N}$, where $\varepsilon$ is the error parameter, $\delta$ is the confidence parameter and $n$ is the length parameter.

2. A may call the procedure example, which returns examples for some concept $f \in \mathcal{F}$, according to an arbitrary and unknown probability distribution $P$ on $X^{[n]}$.

3. For all concepts $f \in \mathcal{F}$ and for all probability distributions $P$ on $X^{[n]}, A$ outputs a concept $g \in \mathcal{F}$, such that with probability at least $1-\delta, P(f \triangle g) \leq \varepsilon$.

As will be clear from the discussion below, the internal behavior of PAC-learning algorithms, i.e. the way in which examples are processed by the algorithms, is of less importance to us. We only require that the output concept is a member of the specified concept class.

Learnability criteria We are interested in the number of examples needed to learn concepts from a class probably approximately correctly. The following complexity measure for learning-algorithms plays an important role.

Definition sample complexity. Let $A$ be a learning algorithm for concept class $\mathcal{F}$. The sample complexity of $A$ is a function $s$ with parameters $\varepsilon, \delta$ and $n$. It returns the maximum number of calls of example by $A$, for all runs of $A(\varepsilon, \delta, n)$, for all $f \in \mathcal{F}$ and all $P$ on $X^{[n]}$. The value of $s$ is infinite if no finite maximum exists.

${ }^{3}$ The symbol $\triangle$ is used to represent the symmetric difference between two concepts. 
Using the above definition, the following learnability criterion for classes can be defined:

Definition PSL. Class $\mathcal{F}$ is said to be polynomial sample learnable (PSL) if there exists a PAC-learning algorithm for $\mathcal{F}$, with a sample complexity that is bounded by some polynomial $p$ in $\frac{1}{\varepsilon}, \frac{1}{\delta}$ and $n$.

Another learnability criterion for classes is that of uniform sample learnability. This notion uses a definition of a PAC-algorithm in which the parameter $n$ is not included. A class is called uniformly sample learnable if there exists an algorithm for it, having a sample complexity that is an integer-valued function in $\frac{1}{\varepsilon}$ and $\frac{1}{\delta}$ only.

Vapnik Chervonenkis dimension It appears ([1]) that a class $\mathcal{F}$ is PSL if and only if it has a polynomial Vapnik Chervonenkis dimension [16]. This is a number associated with a class, which involves the notion of shattering:

Definition shattering. A class of concepts $\mathcal{F}$ on $X$ shatters a set $S \subseteq X$ if the set given by $\{f \cap S \mid f \in \mathcal{F}\}$ is the power set of $S$, denoted by $2^{S}$.

Now, the Vapnik Chervonenkis dimension (VC-dimension) of a class is defined as follows:

Definition VC-dimension. The VC-dimension of a concept class $\mathcal{F}$ on $X$ is the greatest integer $d$ (if it exists) such that there exists a set $S \subseteq X$ of cardinality $d$ that is shattered by $\mathcal{F}$. It is denoted by $\operatorname{D}_{\mathbf{v c}}(\mathcal{F})$.

If for each concept $f$ in $\mathcal{F}, f^{[n]}$ denotes $f \cap X^{[n]}$ and if $\mathcal{F}^{[n]}$ is defined as $\left\{f^{[n]} \mid f \in \mathcal{F}\right\}$, then we say that $\mathcal{F}$ is of polynomial dimension if $\mathbf{D}_{\mathbf{v c}}\left(\mathcal{F}^{[n]}\right)$ is bounded from above by a polynomial in $n$.

The following lemma [10] concerns the relation between the VC-dimension of a class and the number of concepts in it.

Lemma 1. Let $\mathcal{F}$ be a class of concepts on domain $X$. Then

$$
2^{d_{v c}} \leq\left|\mathcal{F}^{[n]}\right| \leq\left(\left|X^{[n]}\right|+1\right)^{d_{v c}}
$$

where $d_{v c}=\operatorname{D}_{\mathbf{v c}}\left(\mathcal{F}^{[n]}\right)$.

So, by finding a tight enough upper bound on $\left|\mathcal{F}^{[n]}\right|$, one can prove that $\mathcal{F}$ has a polynomial VC-dimension from which it would follow that $\mathcal{F}$ is PSL.

Uniform sample learnability has a significant link to the VC-dimension as well. In [1] it is proved that in order for a class to be uniformly sample learnable, it has to have a finite VC-dimension and vice versa. This will come up again in Section 6.

\section{Model Inference}

It appears that PAC-learning theory can be applied to what is known as model inference in first order logic. Specifically, we can consider least Herbrand models of programs as concepts in classes of models, for which the existence of a polynomial VC-dimension can be sought. In this section, we will give some basic notions in model inference. 
Notations In the following, we will denote the predicates in a first order language by $E v e n, P$, etc. Functions will be denoted by $f, g, s$, etc., and variables by a capital $X_{1}, Y$ etc. We will denote constants by lower-case characters $a, b$ or digits $0,1, \ldots$ Also, to represent terms we use $t, t_{1}, t_{2}$ etc., for literals we use $L_{1}, L_{2}$, etc., and for clauses $C, C_{1}, \ldots$

An example We will start off with an example to grasp the intuitive idea of model inference. Consider a first order language $\mathcal{L}$, consisting of the predicate Even, the function $s$ (successor), and the constant 0 . Let $\mathcal{L}_{0}$ be the set of ground (variablefree) atoms of $\mathcal{L}$ and $\mathcal{L}_{h}$ be the set of Horn-Clauses over $\mathcal{L}$. Suppose, that the ground atoms $E v e n(0), \operatorname{Even}(s(s(0)))$, etc. are true and Even $(s(0)), \operatorname{Even}(s(s(s(0))))$, etc. false. Then we would like to find, for example, the following set of clauses in $\mathcal{L}_{h}$ :

$$
\begin{aligned}
& \operatorname{Even}(0) \\
& \operatorname{Even}(s(s(X))) \leftarrow \operatorname{Even}(X) .
\end{aligned}
$$

These clauses imply all true ground atoms and no false ones.

Model inference problem The model inference problem in first order logic is defined as follows ([14]):

Definition model inference problem. Suppose we are given some unknown model $M$ for a first order language $\mathcal{L}$. Can we find a finite set of clauses (a theory), all true in $M$, that imply all true sentences in $\mathcal{L}_{o}$ and no false ones? We call such a theory an $\mathcal{L}_{o}$-complete axiomatization of $M$. The clauses from which the theory is allowed to be constructed are contained in a subset $\mathcal{L}_{h}$ of $\mathcal{L}$, called the hypothesis language.

Remark. In the rest of this article we will, given a first order language $\mathcal{L}$, let $\mathcal{L}_{\text {o }}$ consist of the ground atoms of $\mathcal{L}$ and $\mathcal{L}_{h}$ of the Horn-clauses of $\mathcal{L}$.

\section{PAC-learning and Model Inference}

Classes of concepts in PAC-learning are determined by the way the concepts are represented, e.g. monotone monomials. We say that a program $T$ represents a set $M$ of ground atoms (a concept) if $M=\{\alpha \mid T \vdash \alpha\}$. Now, it is known that any consistent set (program) $T$ of Horn-clauses (!) in language $\mathcal{L}$ has the least Herbrand model, which is unique for $T$. The least Herbrand model for a program $T$ is identified by a set of ground atoms, corresponding exactly with the set of ground atoms that are logical consequences of $T$. So, we can also say that a set of Horn-clauses $T$ represents a set of ground atoms (concept) $M$ if $M$ corresponds with the least Herbrand model of $T$.

Thus, a class of concepts $\mathcal{M}$ in $\mathcal{L}_{0}$ contains sets of ground atoms, such that for each $M$ in $\mathcal{M}$ there is a program $T$ (of some class-specific type) of Horn-clauses having a least Herbrand model that corresponds with $M$. 
Remark. In the following, we will also use the term (Herbrand) 'model' where we actually mean the concept/set of ground atoms corresponding to it. This is to indicate that we are dealing with concepts of ground atoms in first order logic. Similarly, we will speak of classes of models. This should not give rise to confusion considering the above discussion.

PAC-learnability of model classes If we use the class notion defined above, we expect a PAC-algorithm for a model class $\mathcal{M}$ to receive as input examples of a model $M \in \mathcal{M}$, according to some probability distribution $P$ over $\mathcal{L}_{0}$ and output a finite set of clauses in $\mathcal{L}_{h}$ (a program) having a least Herbrand model (also in $\mathcal{M}$ ) such that the PAC-requirements are fulfilled.

Now, suppose that our first order language $\mathcal{L}$ consists of the following symbols:

- predicates $P_{1}, \ldots, P_{p}$.

- functions $f_{1}, \ldots, f_{m}$.

- constants $c_{1}, \ldots, c_{k}$.

Other symbols in atoms in $\mathcal{L}_{o}$ are '(', ')', and ','. Let $K$ denote the total number of symbols: $K=p+m+k+3$ and let the length of an atom $\alpha$ in $\mathcal{L}_{o}$ equal the number of symbols in $\alpha$. Then it can be proved that any consistent model inference algorithm for a class $\mathcal{M}$ over $\mathcal{L}_{o}$, will after $\frac{1}{\varepsilon}\left((n+1) \boldsymbol{D}_{\mathbf{v c}}\left(\mathcal{F}^{[n]}\right) \ln K+\ln \frac{1}{\delta}\right)$ calls of example, output a set of clauses in $\mathcal{L}_{h}$ such that the PAC-requirements are met. Therefore, it is a PAC-algorithm. (By 'consistent' we mean that the algorithm always outputs a concept consistent with the examples seen so far.) The proof of the above statement is analogous to [10] pp. 22-23.

Notice, that if $d=\mathbf{D}_{\mathbf{v c}}\left(\mathcal{M}^{[n]}\right)$ is bounded by a polynomial in $n$, then the number of facts/examples needed is bounded by a polynomial in $n, \frac{1}{\varepsilon}$ and $\frac{1}{\delta}$. In other words (as we saw in Section 1), if $\mathcal{M}$ is of polynomial dimension, it is polynomial sample learnable.

From the following example, a restriction on the type of classes for which PSL is possible follows:

Example. Let $\mathcal{M}$ be the class of all models in some first order language $\mathcal{L}$, representable by a program in a finite number of clauses. Consider $\mathcal{L}_{o}^{[n]}$, the domain of ground atoms of length $\leq n$. Clearly, every subset of $\mathcal{L}_{o}^{[n]}$ has a finite program to represent it, namely a listing of the subset itself. Therefore, it is a member of $\mathcal{M}$. From this, it follows that the whole of $\mathcal{L}_{o}^{[n]}$ is shattered by $\mathcal{M}$. This implies, since the number of ground atoms in $\mathcal{L}_{o}^{[n]}$ is not polynomial in $n$, that the VC-dimension of $\mathcal{M}^{[n]}$ is not polynomial in $n$. Thus, $\mathcal{M}$ is not polynomial sample learnable. Also, if we require the models in a class all to contain a finite number of ground atoms, PSL cannot be achieved.

Thus, it seems reasonable to concentrate on classes of models, representable by a program in some fixed maximum number of clauses. Classes of models representable by programs in some maximum number $c$ of clauses having some maximum number $l$ of literals, for some fixed $c, l$, will from now on be denoted by $\mathcal{M}_{c, l}$. This is the type of classes we will focus on. 
An algorithm In [14], Shapiro suggests an algorithm for model inference, referred to by him as a naive learning algorithm. It is based on arranging all possible theories over a language $\mathcal{L}$ in a sequence, which is possible if we are dealing with a finite number of predicate-, function- and constant symbols in $\mathcal{L}$. As positive and negative examples come by, the current theory is kept as long as it is consistent with the examples seen so far. If it becomes inconsistent, the next theory becomes the current theory etc. So, if we can find a class of models $\mathcal{M}$ of polynomial VC-dimension, then this algorithm learns $\mathcal{M}$ with a polynomial sample complexity.

It should be noted, that Shapiro's algorithm is adapt for models having the property of $h$-easyness, which is, basically, a minor restriction on their complexity and has to do with the number of computational steps within which one can conclude whether a theory is consistent with a set of examples or not. In the rest of this paper we will assume $h$-easyness as an additional property for the discussed model classes, without going into the matter further. For the interested reader, we refer to [14].

\section{How to Prove Polynomial Sample Learnability}

The real challenge after defining the boundaries of the discussion is to actually identify specific classes of concepts as being or not being polynomial sample learnable. All classes we are going to consider will be of the type $\mathcal{M}_{c, l}$, with some additional restrictions regarding the form of the clauses. Now, in order to prove whether or a class $\mathcal{M}_{c, l}$ is PSL, information is needed regarding its VC-dimension. By Lemma 1 this information can be obtained from the inequality $2^{d} \leq\left|\mathcal{M}_{c, l}^{[n]}\right|$, where $d=\operatorname{Dvvc}_{\mathbf{v c}}\left(\mathcal{M}_{c, l}^{[n]}\right)$. We want to find an upper bound on $\left|\mathcal{M}_{c, l}^{[n]}\right|$ of the form $2^{p(n)}$ where $p$ is some polynomial in $n$.

Problems Unfortunately, simply counting the intersections of all models in $\mathcal{M}_{c, l}$ with $\mathcal{L}_{0}^{[n]}$ is out of the question. We need to count the expressions (programs) that have the concepts in $\mathcal{M}_{c, l}^{[n]}$ as their least Herbrand models. Of course we only need those programs structured according to the definition of $\mathcal{M}_{c, l}$. Unfortunately (again!), for every intersection of a model with $\mathcal{L}_{0}^{[n]}$, there will probably still be a vast number of programs all equally qualified to represent it. Including all these programs in establishing our upper bound is very likely to result in disaster.

Equivalence classes Now, consider the following: we call two programs equivalent if they behave the same on $\mathcal{L}_{o}^{[n]}$, i.e. if their least Herbrand models have the same intersection with $\mathcal{C}_{0}^{[n]}$. This gives us an equivalence class of programs for every intersection of a model in $\mathcal{M}_{c, l}$ with $\mathcal{L}_{o}^{[n]}$. What we need, in theory, is the number of equivalence classes. What we will do, actually, is trying to find an acceptably small number of programs for each equivalence class, such that we only have to count programs instead of intersections of models with $\mathcal{L}_{0}^{[n]}$.

Representative program Our hope is that we only have to consider programs of a certain form. In fact, we hope that: if $M$ is a model in $\mathcal{M}_{c, l}$, then $M^{[n]}$ can be 
represented by a program containing only literals of length at most $h(n)$, for some polynomial $h$. In that case we would only have to count the number of programs in our class consisting of literals of less than $n$ characters, which is a relatively easy job. This number would be an upper bound on the number of equivalence classes, which is the number of intersections of models in $\mathcal{M}_{c, l}$ with $\mathcal{L}_{0}^{[n]}$. Formally:

Question. Let $\mathcal{M}_{c, l}$ be a class of models over $\mathcal{L}$ and let $M \in \mathcal{M}_{c, l}$. Suppose that $M$ can be represented by a program $T$. Is there a program $T^{\prime}$ giving the same output as $T$ on examples of length $\leq n$, and containing only literals of length $\leq h(n)$, where $h$ is a polynomial in $n$ ?

Polynomial sample learnability Suppose that this is true for some class $\mathcal{M}_{c, l}$. Now, let $K$ be $p+m+k+3+1$, where $k, m$ and $p$ are defined as in Section 3 (the ' 1 ' is included to count the empty symbol). Then, in a clause of at most $l$ literals of length $\leq h(n)$, there are $l \cdot h(n)$ places to put symbols. We can choose from the $K$ symbols (as above) and variables. The number of different variables in such a clause is limited by $l \cdot h(n)$. So, the number of different clauses of maximally $l$ literals of length $\leq n$ is bounded from above by

$$
\left((K+l \cdot h(n))^{l \cdot h(n)}\right.
$$

The number of programs in clauses of this form is bounded by

$$
\left((K+l \cdot h(n))^{c \cdot l \cdot h(n)}\right.
$$

So, according to Lemma 1, we have:

$$
2^{d} \leq(K+l \cdot h(n))^{c \cdot l \cdot h(n)}
$$

It follows, that

$$
d \leq c \cdot l \cdot h(n)^{2} \log (K+l \cdot h(n))
$$

This means that class $\mathcal{M}_{c, l}$ is polynomial sample learnable.

\section{The Class of Context Free Models}

Consider the following program $T$ :

$$
\begin{aligned}
& P(X, 0, X) . \\
& P(s(X), Y, s(Z)) \leftarrow P(X, Y, Z) . \\
& P(s(s(s(X))), s(Y), s(s(s(Z)))) \leftarrow P(X, Y, Z) .
\end{aligned}
$$

If we want to derive the ground atom $\alpha=P(s(0), 0, s(0))$ from this program by SLD-resolution (essentially, we derive the empty clause from $T \wedge \neg \alpha$ ), then we can see that the third clause will not be used in the process. In fact, this clause will not be used to derive any atom in $\mathcal{L}_{o}$ of smaller length than its positive literal. We can use this kind of property in proving that the classes $\mathcal{M}_{c, 2}$ of context free models are 
PSL. Context free models are models representable by a program, in which every clause is an atom or a two-literal clause of the form

$$
P\left(t_{1}, \ldots, t_{m}\right) \leftarrow P\left(X_{1}, \ldots, X_{m}\right) .
$$

where each variable $X_{i}$ occurs in $t_{i}$. The notion of context free programs was first defined in [13].

Example. As an example, consider the notion of 'plus'. This can be captured by a context free program in a language with 3 -ary predicate Plus, constant 0 and 1-ary function $s$ (the successor function) as follows:

$$
\begin{aligned}
& \operatorname{Plus}(0, X, X) . \\
& \operatorname{Plus}(s(X), Y, s(Z)) \leftarrow \operatorname{Plus}(X, Y, Z) .
\end{aligned}
$$

Size and length Before we formalize the above and give the proof, we will adopt an alternative for the length function. We suggest the use of the size operator:

Definition size. The size of a term in a first order language is the total number of variable-, function-, and constant symbols appearing in it. The size of an atom is the sum of the sizes of the terms it contains as arguments.

As an example, consider the the ground atom $P(s(s(X)), 0)$. The size of this atom is 4 .

It is easy to prove that length and size are equivalent, i.e. in establishing a polynomial VC-dimension for a class, it does not matter whether it is polynomial in the size or in the length of the ground atoms. The set of sentences in $\mathcal{L}_{o}$ of size at most $n$ will be denoted by $\mathcal{L}_{o}^{(n)}$. Analogous notations go for classes and concepts.

We have the following result concerning context free model classes:

Theorem 2. The classes $\mathcal{M}_{c, l}$ of context free models are PSL.

Proof. We will prove that the function $h(n)$, mentioned in the question of Section 4 equals $n$ for this class. Let $M \in \mathcal{M}_{c, l}$ and let $T$ be a program having $M$ as its least Herbrand model. Let $\alpha$ be any ground atom in $M$, with $\operatorname{size}(\alpha) \leq n$ for some $n$. Since $M$ is the least Herbrand model of $T$ and $\alpha$ is in $M$, it is known, by the refutation completeness of SLD-resolution, that there is a finite derivation of the empty clause from $\{\neg \alpha\} \cup T$ by SLD-resolution. Now, $\alpha$ cannot be unified with a positive literal in $T$ of size $>n$. If $\alpha$ can be unified with the positive literal $L$ from clause $C=\left(L \leftarrow L_{1}\right)$ by substitution $\sigma$, then

1. $L_{1} \sigma$ will be ground, since all variables of $L_{1}$ are in $L$ and they are instantiated by ground terms according to $\sigma$.

2. The size of $L_{1} \sigma$ will not exceed $n$. This follows from the fact that in negative literals no function symbols or constants occur and each variable in $L_{1}$ occurs in its corresponding term in $L$. 
So, in the next resolution step, where we derive $L_{1} \sigma$ from $T$, we have a similar situation as in deriving $\alpha$ itself. Again no unification can take place with any positive literal of size $>n$, and after unification with some positive literal, the negative literal (if present) will, after substitution, not have size $>n$. It follows that in the resolution procedure for $\alpha$, the clauses in $T$ containing literals of size $>n$ will not be needed. If we delete from $T$ all such clauses, we are left with a program $T^{\prime}$, equivalent to $T$ with regard to the atoms in $\mathcal{L}_{o}^{(n)}$, containing only literals of size $\leq n$. This is true for every model in $\mathcal{M}_{c, l}$ and for every $n$. It follows that $h(n)=n$ for this class. Thus, $\mathcal{M}_{c, l}$ is PSL, by the arguments used in Section 4.

Shapiro Recall Shapiro's suggestion for an implementation of a model inference algorithm. This can become a PAC-algorithm for the above class, having a polynomial sample complexity. To make it suitable for this class the only adaptation needed is having the sequence of all possible theories in $\mathcal{L}$ replaced by the sequence of all possible theories within the context free class. This argument is valid for every class of models that is PSL.

\section{$6 \quad$ Another Learnable Class}

The class of context free models is significant, but has limited possibilities. We want to generalize it to some extent, such that the arguments used in the previous section to prove PSL, are still valid. We have arrived at: the class $\mathcal{M}_{c, l}$ for some fixed $c, l$, with the additional requirement, that in any clause $C=\left(L \leftarrow L_{1}, \ldots, L_{m}\right)$ of a program in $\mathcal{M}_{c, l}$, any argument in one of the $L_{i}$ must be contained in an argument in $L$.

Example. A program in this class will contain clauses of the form:

$$
P(f(g(X)), f(Y)) \leftarrow Q(g(X), f(Y), Y), R(f(g(X))) .
$$

every argument on the right of the ' $\leftarrow$ ' is a subterm of an argument in the positive literal.

Theorem 3. Class $\mathcal{M}_{c, l}$ is polynomial sample learnable.

Proof. Let the maximum arity of all predicates in $\mathcal{L}$ be $A$. We will prove that for the class $\mathcal{M}_{c, l}, h(n)=n A$, where $h$ is again the function from the question in Section 4. We can use arguments similar to those used for the context free models. Suppose that we have a program $\mathcal{T}$ for some $M \in \mathcal{M}_{c, l}$ and a ground atom $\alpha$ in $M$ of size $\leq n$ for some $n$. Notice that all terms in $\alpha$ must be of size $\leq n$. If we want to derive a contradiction from $\neg \alpha \wedge T$ then $\alpha$ cannot be unified with positive literals containing terms of size $>n$. So $\alpha$ will not unify with any positive literal of size $>n A$. Also, if $\alpha$ unifies with positive literal $L$ in clause $C=\left(L \leftarrow L_{1}, \ldots, L_{m}\right)$ by substitution $\sigma$, then any negative literal $L_{i}$ in $C$ will, after substitution, be ground and not contain terms of size $>n$. This is because for all $i$ the terms in $L_{i} \sigma$ will be subterms of terms in $L \sigma$. It follows that the size of $L_{i} \sigma$ will not exceed $n A$. The same arguments are valid if we want to derive $L_{i} \sigma$ from $T$. It follows that in deriving $\alpha$ 
from $T$, clauses with literals containing terms of size $>n$ will not be needed. Thus, by deleting such clauses from $T$, we have a program $T^{\prime}$, equivalent to $T$ with regard to $\mathcal{L}_{0}^{(n)}$, containing only terms of size $\leq n$, so every literal has size $\leq n A$. This can be done for all $M \in \mathcal{M}_{c, l}$ and all $n$. It follows that $h(n)=n A$ for this class and thus $\mathcal{M}_{c, l}$ is PSL.

Remark. In the above class, we can relax the restrictions regarding constants, appearing in a program in $\mathcal{M}_{c, l}$ slightly, while preserving PSL. There are two possibilities: the first is to require that in any clause, the constant term of the largest size always appears in the positive literal. Notice that this does not imply that any constant term in a negative literal is contained in a term in the positive literal. The second option is to restrict the maximum size of any constant term appearing in a program. In both cases, the function $h(n)$ will still be bound by a polynomial in $n$.

\section{Uniform Sample Learnability of Model Classes}

We have proved the fact that the class $\mathcal{M}_{c, l}$ of context free models is PSL. In the introduction we also mentioned uniform sample learnability. Now, uniform sample learnability is a more stringent complexity criterion than PSL. Is the class of context free models uniformly sample learnable? From the following, we can see that it is not.

The interesting classes In this section we will briefly discuss uniform sample learnability of model classes. We will prove that most of the 'interesting' classes of models are not uniformly sample learnable. As was stated in Section 1, this can be done by proving that these classes do not have a finite VC-dimension.

The classes we are particularly interested in are again those classes containing models representable by a program in at most $c$ clauses of at most $l$ literals, for some fixed $c, l$.

Now, let $\mathcal{L}$ be a language containing at least one predicate $P$, one function $f$ and one constant $a$. then:

Proposition 4. $\mathcal{M}_{\mathfrak{c}, l}$ for $\mathcal{L}$ is not uniformly sample learnable, for all $c, l>1$.

Proof. We will show that $\mathcal{M}_{2,2}$ does not have a finite Vapnik Chervonenkis dimension and is therefore not uniformly sample learnable. From this it follows that every superclass of this class (i.e. a class with greater $c$ and/or $l$ ) is also not uniformly sample learnable.

Suppose $P$ and $f$ are of arity 1 . We will denote an $n$ times nested function by $f^{n}$, e.g. $f(f(f(a)))$ is denoted by $f^{3}(a)$. Now, if we want anything in $\mathcal{L}_{o}$ to be deducible from a program of 2 clauses of maximally 2 literals, then it has to contain at least one one-literal clause. Consider the situation where this clause is ground and that the other one is a two-literal clause containing a variable, say $X$. Let $x_{1}=210$, $x_{2}=330, x_{3}=462, x_{4}=770, x_{5}=1155$. Notice, that every $x_{i}$ is the product of 4 out of the 5 first prime integers: $2,3,5,7,11$. Now consider the following set $S$ of ground atoms: $\left\{P\left(f^{x_{1}}(a)\right), P\left(f^{x_{2}}(a)\right), P\left(f^{x_{3}}(a)\right), P\left(f^{x_{4}}(a)\right), P\left(f^{x_{5}}(a)\right)\right\}$. Suppose 
that model $M$ contains $P\left(f^{x_{2}}(a)\right), P\left(f^{x_{4}}(a)\right)$ and $P\left(f^{x_{5}}(a)\right)$ but not $P\left(f^{x_{1}}(a)\right)$ and $P\left(f^{x_{3}}(a)\right)$. Consider the following program:

$$
\begin{aligned}
& P(a) . \\
& P\left(f^{55}(X)\right) \leftarrow P(X) .
\end{aligned}
$$

From this program, all three true atoms can be derived and no false one. The reason for this is that 55 is the greatest common divisor of $x_{2}, x_{4}$ and $x_{5}$, and is the product of the two primes 11 and 5 by which resp. $x_{1}$ and $x_{3}$ are not divisible. Actually, we can construct a similar program for every mapping of the five ground atoms to $\{$ true, false $\}$ : we just replace the number 55 by the greatest common divisor of the $x_{i}$ 's of the true atoms. Therefore, such an $S$ is shattered by $\mathcal{M}_{2,2}$.

Indefinitely larger shattered sets of ground atoms can be constructed, if we increase the number of $x_{i}$ 's by using more consecutive primes. Therefore, the Vapnik Chervonenkis dimension of even this simple class is not finite. We only have to modify the proof slightly if we want it to be valid for classes over languages in more predicates and functions having larger arity as well. Now, since this class is a subclass of every class described in the proposition, the validity of the proposition follows immediately.

As we can see, the programs constructed to prove non-uniform sample learnability are all context free. From this it follows that, as was mentioned before, the class $\mathcal{M}_{c, l}$ of context free models is not uniformly sample learnable.

\section{Conclusions}

In this paper we have attempted to combine PAC-learning with model inference. The second can be seen as a subject in the field of Inductive Logic Programming. Looking upon Herbrand models as concepts in the domain of all ground atoms enables us to consider the VC-dimension of classes of models. We can find an upper bound on this dimension by counting the number of different programs fit to represent the models in a class restricted to $\mathcal{L}_{0}$. For two classes, among which the class of context free models, this upper bound is tight enough to establish PSL. Uniform sample learnability, which is a stronger requirement than PSL, is out of the question for the more 'interesting' classes.

Open Problems Frankly, all additional restrictions on $\mathcal{M}_{c, l}$-type classes bother us: we would like to drop them without losing PSL. However, consider the following program.

$$
\begin{aligned}
& P(s(s(s(a)))) . \\
& P(X) \leftarrow P(s(X)) .
\end{aligned}
$$

If we want to derive $P(s(s(a)))$ from this program then the first clause will be used: this clause cannot be omitted to get a program equivalent with regard to atoms of size $\leq 3$. We have similar problems when allowing for variables in the body of a 
clause that do not appear in the head. So, in proving PSI for more general classes than the ones described above, the old arguments are no longer valid. New proof methods will have to be found.

Furthermore, interesting problems arise when considering the so-called one-sided learnability of model classes, which means that one only allows mistakes in the output of a specific type: inclusion-only errors or exclusions-only errors. This has a significant link to the question, whether or not the intersection (or union) of two models in a class, is a model in the class as well. Our expectation is that the intersection of two context free models in a class $\mathcal{M}_{c, l}$ is a model in this class as well.

\section{Related Work}

Applying the PAC-learning paradigm to classes of logic programs is a subject that has received considerable attention in the last few years. A lot of authors doing research in this area discuss the subject of polynomial time learnability, which involves the number of computational steps needed to PAC-learn a class of concepts, thus implicitly including sample learnability (polynomial sample learnability, which we discuss as a separate issue, is a prerequisite for polynomial time learnability), the consistency problem and the evaluation problem. The consistency problem concerns the number of steps to compute a logic program (in some class) consistent with a set of examples, and evaluation problem concerns the number of steps to classify an example using some output theory. We will give a brief overview of some of the results in these areas.

In [8] the idea of feeding PAC-learning algorithms for model classes with 'good' data to speed up convergence, is proposed. In this way, the quality of the examples, which is believed to be essential in human learning, is included in the paradigm. Indeed, positive learnability results can be achieved if the requirement of random examplegeneration is replaced by so-called generative representative sets of examples.

In [11] the approach is to have a learning algorithm, given some fixed background theory $T$, generate one sentence $S$, such that $T \cup S$ will behave similarly to $T \cup S^{\prime}$ ( $S^{\prime}$ being the 'target' sentence). The term 'similarly', of course, can be filled in to contain PAC-requirements. It is proved, that under certain restrictions, the class of constrained atoms is polynomial time learnable. Constrained atoms are Horn clauses in which each variable in the body also appears in the head of the clause, which was also the case in the classes for which we proved PSL.

In [4], it is shown that the class of $i j$-determinate Horn clauses is polynomial time learnable, where an $i j$-determinate Horn clause is a clause in which the body can contain variables not appearing in the head under the restriction that these variables have a unique binding, that the so-called depth of the clause is bounded by a constant ( $i$ ) and that the arity of the body literals is bounded by a constant $j$. These clauses are in some respects more general than those in the program classes we studied, because new variables may appear in the body of a clause. However, the results are still concerned with learning one clause. PAC-learnability of non-recursive, functionfree programs of maximally $c i j$-determinate Horn clauses, for some constant $c$, can be proved under the condition of so-called 'simple distributions'. 
Very recently, Cohen [2], proved PAC-learnability results for a number of classes, among which are also several one-clause program classes. It is proved that log-depth (instead of constant depth) j-determinate clauses are not polynomial time learnable. The class of non-recursive, function-free programs in any number of clauses with a maximum of $l$ literals in the body is proved to be polynomial time learnable from negative examples only. It is also shown that allowing for recursion in the programs of a class introduces severe learnability problems. These can be (partially) solved by allowing only for so-called linear recursion, where only one literal in the body of a clause is allowed to cause recursion (see also [3]).

In [7] some negative learnability results are proved. It is shown that dropping the requirement of $i j$-determinacy leads to non-polynomial time learnability. Dropping only the fixed depth requirement or only the determinacy requirement will also render non-polynomial time learnable classes.

Finally, in [5] it is shown that the class of programs of maximally $c$ clauses (for some constant $c$ ), which are constrained, non-recursive and function free, is polynomially learnable.

As the reader will have noticed, most research in this area is aimed at finding efficiently learnable model classes with as much expressive power as possible. However, polynomial time learnability, as a criterion, imposes quite stringent restrictions of the definition of learnable classes. In concentrating solely on sample learnability, thus to some extent ignoring the consistency problem, these restrictions can be partly removed. The most important issue then becomes a polynomial VC-dimension, a topic we did not find discussed explicitly very often in work about classes of logic programs.

A lot of positive results in polynomial time learnability do, however, involve classes of programs in which the requirement of constraind-ness is replaced by bounded depth or determinacy. This is an interesting topic that we have not addressed so far.

\section{References}

1. Blumer, A., Ehrenfeucht, A., Haussler, D., Warmuth, M.: Learnability and the VapnikChervonenkis Dimension. Journal of the Association for Computing Machinery 36 No. 4 (1989) 929-965

2. Cohen, W.: Learnability of Restricted Logic Programs. Proceedings of the Third International Workshop on Inductive Logic Programming (ILP'93) (1993) 41-71

3. Cohen, W.: PAC-Learning a restricted Class of recursive Logic Programs. Proceedings of the Third International Workshop on Inductive Logic Programming (ILP'93) (1993) 73-86

4. Dzeroski, S., Muggleton, S., Russel, S.: PAC-Learnability of Determinate Logic Programs. Proceedings of the Fifth ACM Workshop on Computational Learning Theory (1992) 128-135

5. Dzeroski, S., Muggleton, S., Russel, S.: Learnability of Constrained Logic Programs. Proceedings of ECML'93 (1993) 342-347

6. Ehrenfeucht, A., Haussler, D., Kearns, M., Valiant, L.: A General Lower Bound on the Number of Examples Needed for Learning. Information and Computation 82 (1989) 247-261

7. Kietz, J.: Some Lower Bounds for the Computational Complexity of Inductive Logic Programming. Proceedings of ECML'93 (1993) 114-123 
8. Ling, X.: Inductive Learning from Good Examples. Proceedings of IJCAI'91 (1991) 751-756

9. Muggleton, S.: Inductive Logic Programming. Muggleton S. (ed.), Inductive Logic Programming, Academic Press (1992) 3-27

10. Natarajan, B.: Machine Learning, a Theoretical Approach. Morgan Kaufman Publishers, Inc (1991)

11. Page, C.D., and Frish, A.M.: Generalization and Learnability: A study of Constrained Atoms. Muggleton (ed.), Inductive Logic Programming, Academic Press (1992) 29-61

12. Polman, M., Nienhuys-Cheng, S.-H.: PAC-Learning and Model Inference. Benelearn '93, Collection of work reports, Vrije Universiteit Brussel (1993)

13. Reynolds, J.C.: Transformational Systems and the Algebraic Structure of Atomic Formulas. Machine Intelligence 5 (1970) 135-153

14. Shapiro, E.: Inductive Inference of Theories from Facts. Technical Report 192, Dept. of Computer Science, Yale University, USA (1981)

15. Valiant, L.G.: A Theory of the Learnable. Communications of the ACM 27 No. 11 (1984) $1134-1142$

16. Vapnik, V., and Chervonenkis, A.: On the uniform convergence of relative frequencies of events to their probabilities. Theory of Probability and its Applications 16 No. 2 (1971) 264-280 